\title{
El camino y el caminar: fuentes históricas de los rarámuri de la Sierra Tarahumara, México
}

DOI

https://dx.doi.org/10.11606/ 1678-9857.ra.2021.184479

\section{María Isabel Martínez Ramírez}

A lo largo de este artículo muestro que, para los rarámuri o tarahumaras que residen en el Norte de México, los caminos y el caminar constituyen un contexto epistemológico caracterizado por revelar los procedimientos necesarios para producir y transmitir contenidos concretos. El objetivo de este escrito es exponer las narrativas recopiladas en dicho contexto para examinar la relación entre la enunciación y los soportes geográficos que son reconocidos como evidencia de los hechos relatados y como generadores de la enunciación. La meta es analizar los procedimientos nativos de la enunciación con el fin de postular una simetría entre estos registros y las fuentes documentales de carácter burocrático y administrativo que el Estado mexicano ha producido durante los siglos XX y XXI y que han servido como un pilar para construir la historia de/sobre los rarámuri. El objeto ulterior es aportar a la construcción de un espacio de existencia para la historia rarámuri.

Rarámuri, historia amerindia, fuentes históricas, registros territoriales 


\section{INTRODUCCIÓN}

Para los rarámuri o tarahumaras - pueblo que, para el 2015, fue censado por el índice lingüístico en aproximadamente 74.000 personas ${ }^{-1}{ }^{1}$ el camino y el caminar trascienden el acto de andary constituyen los fundamentos de sus proyectos políticos y modos de existencia, tal como fue reiterado por la etnografía, la historia y la literatura durante el siglo XX (Kennedy, 1970; Merrill, 1988; Montemayor, 1999 [1995]; Sariego Rodríguez, 2015 [2012]; Fujigaki Lares, 2019). Los rarámuri son conocidos por ser grandes corredores, como la maratonista internacional Lorena Ramírez, y por residir de manera extensiva en pequeñas rancherías a lo largo y a lo ancho de la Sierra Tarahumara que, conformada por un macizo montañoso de la Sierra Madre Occidental, cruza el estado de Chihuahua en el norte de México. Por ello, la movilidad realizada entre las residencias habitacionales y de cultivo ubicadas en las altas cumbres (hasta $2400 \mathrm{msnm}$ ) y en las profundas barrancas (hasta 200 msnm) constituye la relación primordial con su mundo, la Sierra Tarahumara o kawí, que simultáneamente los conforma como personas. ${ }^{2}$

Como Catarino de Coyoachique, en el municipio de Urique, le compartiría a S. Aguilera Madrigal (2011: 35): "todo tiene su camino [...], las aguas, el sol, las nubes, las flores y demás. Vivir en armonía significa seguir este camino marcado por Dios - pues éste fue recorrido y marcado primero por Tata Riosi, para que después los ralámuri [sic] supieran por dónde caminar". Los cuerpos rarámuri están compuestos por una red interna de caminos de sangre (laá boára). Las almas (llamadas regionalmente de forma diferente: alewá, arewáka, iwigá) transitan por ellos para generar movimiento, calor, pensamientos, sentimientos, acciones y estados de salud. Éstas se organizan como familia o como grupo de autoridad y habitan el cuerpo como si se tratara de una casa. En ocasiones, algunas salen para viajar por sendas oníricas, y se extravían cuando son raptadas, a causa del susto, o simplemente al perder el camino de vuelta a su residencia corporal. Para recobrar estas almas, los-que-saben-curar o doctores (owirúame) seguirán las huellas del enfermo y negociarán su regreso, caminarán en la dimensión onírica, realizarán un ritual, un sacrificio y una danza, ya que danzar es caminar en el plano celeste (Montemayor, 1999 [1995]: 26). Los caminos también son aquellos que Onorúame (El-que-es-Padre) y Eyerúame (La-que-es-Madre) - ocasionalmente identificados con el astro solar y con la luna - recorren durante su andar por la cúpula celeste y que enseñaron a los antepasados de los rarámuri (anayáwari) bajo la forma de la costumbre (anayáwari boé, literalmente el camino de los antepasados). En conjunto, estos senderos deben ser transitados colectivamente. Así, los rarámuri caminan cotidianamente para visitar amigos y parientes con el fin de multiplicar y de cuidar los lazos necesarios para ser un rarámuri, pues cada uno de estos andares conforma los senderos internos de las personas. En este mundo se hace camino siendo y haciéndose caminos.

Por todo esto, como expongo en este escrito, los caminos y el caminar constituyen un contexto de producción y de transmisión del conocimiento sobre aquello que
1 | Estimación del INALI con base en los datos de la Encuesta Intercensal, INEGI, 2015, y el Cátalogo de las Lenguas Indígenas Nacionales, INALI, 2008 , https://site.inali.gob. $\mathrm{mx} /$ Micrositios/ estadistica basica/estadisticas2015/pdf/ agrupaciones/tarahumara.pdf [consulta: 14 de junio de 2018].

2 | Los rarámuri utilizan el vocablo kawí para remitir a su mundo, por ejemplo en la frase inicial de algunos relatos, tales como "el mundo se formó en tres épocas" (kawí ko, a bikiná kayáame niráa kayénali) (Gardea y Chávez, 1998: 20); o para referir a la Sierra Tarahumara, como en las recientes declaraciones del gobernador de Choréachi, Macario Ramos, en torno a la explotación del bosque. Para escuchar declaraciones del gobernador de Choréachi, Macario Ramos, en torno a la explotación del bosque, ver https://soundcloud. com/chor-achi-rar-muri [consultado 13 abril 2019]. De la misma manera, los spots de radio de Voces de la Sierra Tarahumara que, en 2015 describían la situación de la construcción del aeropuerto en Creel y el conflicto agrario en e ejido de San Elías Repechique inician su presentación con la siguiente frase: rarámuri kawi gára ra'ichára, literalmente "hablantes de rarámuri de la Sierra Tarahumara" o "voces de la Sierra Tarahumara". Voces de la Sierra. Contec. Consultoría Técnica Comunitaria A.C., 2016 [consultado 7 marzo 2016]. Disponible en http://kwira.org 
los rarámuri de Munérachi, Batopilas, llaman chabé nilúame natáli (literalmente "lo que se piensa antes") (Moreno Pineda, 2013: 148), esto es, sobre los relatos contados desde tiempos antiguos y el conocimiento enseñado por los antepasados (anayáwali). Debemos a K. H. Basso (1996) una etnografía emblemática sobre la relación entre el lenguaje y el paisaje (landscape) para algunos apaches de Cibecue en el sur de los Estados Unidos. Al igual que para el caso rarámuri, los "place-worlds" que producen y reproducen el conocimiento sobre el pasado son las personas (Basso, 1996: 18); en otras palabras, el paisaje influye en la autodefinición de los apaches y de la acción social (Basso, 1996: 65). A diferencia de F. Santos-Granero (1998: 131, 132), quien se interesó en dar cuenta de la codificación de una historia "escrita" en el paisaje mediante una "escritura topográfica" en el territorio yanesha de Yorenaco en los Andes Centrales de Perú, Basso enfocó su atención en los modos de transmisión del conocimiento y en las condiciones particulares de la enunciación, ambos temas afines a mis intereses. Como muestro a lo largo de este texto, en contraste con el material recopilado por F. Santos-Granero: S. Hugh-Jones (2016: 165) interesado en cómo los petroglifos, las rocas y los rápidos de los ríos no sólo eran materializaciones de la historia sino modos de pensar sobre el tiempo en el Alto Río Negro; y la investigación de D. Paiva Ramos (2013) realizada en la misma región y caracterizada por elaborar una original etnografía del andar; para los rarámuri que conocí en la Sierra Tarahumara los sitios vinculados con una narrativa podían ubicarse simultáneamente en espacios distintos y lejanos entre sí. Era común que en cada región existiera un sitio llamado Ganóchi (lugar de gigante) asociado con relatos sobre gigantes; o bien una o varias cuevas, relacionadas con alguna historia sobre apaches que lloraban, denominadas Narárachi (lugar de llanto). Como advirtió A. Fujigaki Lares (2015), este también era el caso de los patios rituales (awírachi), ya que una vez preparado el espacio mediante la limpieza y el aplanado de la tierra suelta, cualquier sitio podía fungir como un awírachi. Echando mano de la jerga de Basso, el sitio-mundo que daba sentido a la proliferación de estos lugares era el kawí o mundo rarámuri, identificado con la Sierra Tarahumara. Dicho de otra manera, los lugares-mundo ubicados dentro del kawí eran espacios virtuales que potencialmente podían actualizarse en cualquier sitio que cumpliera con ciertos requisitos (ser aplanado, ser una cueva con marcas de fuego, ser una roca gigante etc.). Como indicó S. Hugh-Jones (2016: 161) para el Alto Río Negro, las geografías de los rarámuri que estaban articuladas con la memoria y con los viajes también se encontraban en el ritual, en el canto y en el sueño.

En este marco, el objetivo de este artículo es exponer las narrativas de algunos rarámuri bilingües recopiladas en español, en el contexto de los caminos y durante el acto de caminar, para examinar la relación entre la enunciación y aquello que denominaré registros territoriales. ${ }^{3}$ Es decir, los soportes geográficos que, desde la perspectiva rarámuri, son reconocidos como evidencia de los hechos relatados y como generadores de la enunciación. La meta es analizar los procedimientos nativos de la enunciación
3 | La lengua tarahumara o rarámuri raíchari pertenece a la familia yuto-azteca. El Equipo de Lingüística de la Oficina de Estudios Especiales de la Coordinación Estatal de la Tarahumara, dependiente del estado de Chihuahua, en 1992 y 1997, reconoció cinco áreas dialectales con base en criterios fonológicos, elementos léxicos y componentes sintácticos. Los materiales presentados en este artículo corresponden al área norte, donde se ubican los municipios de Bocoyna, Guachochi, Urique y Carichí, con Sisoguichi y Narárachi como comunidades representativas. Aproximadamente 74.000 personas hablan rarámuri raî́chari, tendencialmente dominan el español o lo comprenden (Islas Flores 2015: 14-17) y, en ciertas regiones, por el contacto con pimas, warijíos, pima-tarahumaras y tepehuanos del norte, los rarámuri son trilingües (de la Parra 2012: 282). Ante la diversidad de escenarios lingüísticos, sugiero revisar e recuento y el estudio de caso presentado en la reciente tesis de Nuvia de los Ángeles Betancourt Sabatin (2020). 
- reconocimiento, lectura y validez. En consonancia con las reflexiones críticas sobre la relación entre la historia, la etnografía y la historia amerindia de P. Cow (2001; 2006), el fin es proponer que dichos registros son simétricos, en el sentido de B. Latour (2001 [1991]), a las fuentes documentales de carácter burocrático y administrativo que el Estado mexicano ha producido durante los siglos XXy XXI y que han servido como un pilar para construir la historia de/sobre los rarámuri. El objeto ulterior es aportar a la construcción de un espacio de existencia para la historia rarámuri y, en esa medida, colaborar con el diálogo entre esta historia amerindia y la historiografía de/sobre los indígenas.

La reflexión que presento en este artículo es producto de la confluencia de dos ejes de indagación. Por una parte, de la revisión bibliográfica dedicada a las fuentes históricas amerindias tales como el cuerpo, el territorio, la danza, la enfermedad, entre otras. Y, por otra, de la preocupación teórico y metodológica para contribuir al espacio de existencia de las historias amerindias. Este proyecto se inspira en las apuestas tempranas de P. Cow (2001: 8-9) quien, con base en su trabajo de campo entre 1980 y 1990, con los piro de Santa Clara en la Amazonía peruana, afirmó que, para los integrantes de este pueblo, su historia no se reducía a nuestra historia en común ("our common history"), esto es, a ese campo caracterizado por la colonización, la globalización y el desarrollo de la economía mundial. Al tomar en serio aquello que los nativos del Bajo Urubamba decían y hacían sobre su historia, P. Gow (2006: 197) develó que el parentesco para los piro era la historia, así como la venganza para los tupinambá (Taylor, 1993) o el paisaje para los apaches (Basso, 1996).

El trabajo de M. Carneiro da Cunha también ha sido estimulante para examinar el espacio de posibilidad de estas historias nativas y de los proyectos políticos y de existencia que las acompañan. Esta antropóloga e historiadora afirmó que, para el caso de Brasil, "queda aún por hacer una historia propiamente indígena. No solamente es el obstáculo real [...] por la ausencia de escritura y por tanto de la autoría de los textos, no es solamente la fragilidad de los testimonios materiales de esa civilización [...], sino la dificultad de adoptar ese otro punto de vista sobre una trayectoria de la que hacemos parte" (Carneiro da Cunha, 1992: 21-22, el resaltado es mío). Para esta autora, el derecho a la recuperación de una historia propia es fundamental en la medida en que la tenencia de las tierras indígenas en Brasil, tal como en México, derivan de una situación histórica. Aún así, desde su perspectiva, no es suficiente comprobar con documentos escritos la ocupación prolongada de los territorios - documentos que, destacó esta etnóloga, en la mayoría de los casos, tienen intereses antagónicos a los de los indígenas. Por el contrario, la meta es reanudar la importancia de estas historias nativas mediante otras fuentes y, como antropólogos, historiadores, ciudadanos y miembros de un Estado nación, reubicar nuestra posición de enunciación con el fin de multiplicar las narrativas sobre nuestro papel en esta historia compartida tal como expusieron D. Kopenawa y B. Albert en A queda do céu. Palavras de um xamã yanomami (Kopenawa y Albert, 2015 [2010]). 
En última instancia, la finalidad de este artículo es aportar un ejemplo de los contextos de producción enunciativa rarámuri con el fin de que las categorías y las prácticas nativas problematicen las premisas epistemológicas que, pese a los vastos debates sobre el tema (ver Cow, 2006), continúan fundamentando las discusiones académicas en torno a díadas como historia/mito, historia/historia oral, historia/ etnohistoria. Desde mi perspectiva, uno de los presupuestos generales de estas distinciones radica en el potencial que el analista otorga a cada una de las fuentes para reconstruir procesos, eventos o hechos verificables -implícitamente definidos como reales. En concordancia con el reciente proyecto de F. Navarrete Linares (2018: 18-19) sobre la historia mexica, con este texto busco poner en marcha la "imaginación histórica" que, promovida por este autor, busca crear "una nueva manera de construir las verdades históricas a través del diálogo entre mundos diferentes". Al igual que este historiador y antropólogo, mi premisa consiste en "suspender nuestros juicios sobre lo que es verdadero y real en los mundos históricos [...] para que cuestionemos eso que llamamos realidad histórica y que en verdad no es más que otro mundo histórico particular" (Navarrete Linares, 2018: 21).

Para lograrlo, primero describo tres contextos de enunciación rarámuri: los consejos, los mitos y la narración colectiva de chabé nilúame natáli (literalmente "lo que se piensa antes"). La meta es ofrecer un panorama mínimo de los contextos de enunciación sobre los sucesos pasados con el objeto de ubicar la producción y la transmisión del conocimiento articulado con el camino y con el caminar. Posteriormente, describo etnográficamente este contexto de enunciación para finalizar con una reflexión sobre por qué los registros territoriales son una fuente de la historia rarámuri.

\section{PALABRAS RARÁMURI: CONSEJOS, SERMONES,}

\section{MITOS Y ENUNCIACIÓN COLECTIVA}

Mis primeros pasos junto a los rarámuri se remontan al 2002. Para mis interlocutores, caminar también era experimentado como un acto pedagógico y, por tanto, epistemológico. Entre mis deberes como estudiante de licenciatura, requería recopilar "mitos", concepto que problematizo adelante. Inicialmente mis expectativas no fueron cubiertas porque la palabra, para los rarámuri que conocí en el ejido de Norogachi, municipio de Guachochi, era un acto análogo al pensamiento y a la acción y, como tal, era un expresión de las relaciones que constituían a cada persona y que la vinculaban con otros existentes y con el mundo (Merrill, 1988: 62-64; Moreno Pineda, 2013: 51-52; Fujigaki Lares, 2015: 351, 357; Rodríguez López, 2017). Además de ser meditada previamente, la expresión de la palabra requería cierta ecuanimidad emocional y mesura.

Una expresión de la particularidad y de la relevancia de la palabra para los rarámuri son las prácticas denominadas en español bajo el término "consejos", tema 
destacado en la etnografía regional (Merrill, 1988; Bonfiglioli, 2005; Vallejo Narváez, 2015). De acuerdo con los rarámuri, Onorúame otorgó el consejo a los antiguos rarámuri (anayáwari) para que configuraran su actual modo de vida. Este consejo ha sido transmitido de generación en generación a través de la palabra y de recorrer colectivamente "el camino de los antepasados" (anayáwari boé), es decir, de practicar la costumbre mediante acciones, pensamientos y palabras en el día a día, así como a través del trabajo colectivo agrícola y ritual. El legado del consejo de Onorúame es transmitido de mayores a menores en dos sentidos. Primero, en los sermones que las autoridades rarámuri ofrecen en las reuniones dedicadas al trabajo y al consumo de cerveza de maíz (teswino o batári), llamadas teswinadas; en los juicios; cada domingo o día de fiesta; así como en los consejos de las personas denominadas mayoras. Segundo, a través del consejo transmitido al interior del grupo residencial.

Entre 2002 y 2012, los rarámuri de Norogachi contaban con tres gobernadores rarámuri o siríame, doce generales, doce capitanes y doce mayoras - jerarquía que, siguiendo a C. W. Pennington (1996 [1963]: 278), fue implantada por los jesuitas durante el siglo XVII. Los siríame - según la declaración del gobernador rarámuri en turno, Antonio Sandoval, en 2006 - estaban encargados de aplicar la justicia, de supervisar la correcta realización de las fiestas y de la continuidad de las costumbres. Sus medios de acción eran los juicios públicos, la coordinación de las fiestas celebradas en Semana Santa y en invierno (Bonfiglioli, 1995), el sermón de cada domingo después de las misas católicas y el consejo personal. Los mayora, por su parte, tenían la tarea de aconsejar a los niños y a las mujeres, así como aquellos que deseaban concretar nuevas uniones conyugales.

Las almas de los towí (niños) y de las tewé(niñas) crecían a la par que el cuerpo, en gran medida, por la incorporación de estos consejos. Por ello, al interior de los grupos residenciales, los consejos se transmitían bajo la misma lógica. En este caso, existía una red extendida de personas mayores capaces de contribuir en esta maduración anímica: padre (tata), madre (nana, chichi), hermanos y hermanas mayores (bachíra, kochíra, bonirá, wayéra), hermanos y hermanas del padre y la madre (kumuche, so'rorá, ri'chirá, ráte, aporá, kulíchi), hijos de los hermanos y hermanas del padre y la madre (bachíra, kochíra, bonirá, wayéra), abuelos paternos y maternos (apalochi, u’sú, akáchuri, ochípari), hermanos de los abuelos paternos y maternos (apalochi, u'sú, akáchuri, ochípari) - para una descripción de esta terminología, ver M. I. Martínez Ramírez, ]. Martínez Galván y N. Naranjo Mijangos (2012: 93-104).

W. Merrill (1988: 61-62), quien realizó una etnografía detallada sobre los sermones, indicó que éste era el contexto de enunciación rarámuri más elaborado en la región de Rejogochi, municipio de Bocoyna. Para este autor, el propósito de estas palabras era reiterar "consejos probados con el tiempo para la conducta apropiada en la vida", y no comunicar información nueva. Nawésari y nátiri son los términos utilizados para referirse al sermón dominical, así como a cualquier tipo de consejo. Ambos sustantivos comparten 
la raíz na- que, en palabras de $W$. Merrill, remite a pensamientos "bien elaborados y opiniones cuidadosamente consideradas" (Merrill, 1988: 62). Para A. Fujigaki Lares (2015: 350-358), el sustantivo nátari enlaza, bajo una lógica explícitamente anti-cartesiana, el corazón, el pensamiento, la palabra y la acción; en otros términos, pensar es actuar. Por ello, en Norogachi, tal como en Rejogóchi, la capacidad retórica era una expresión de la fuerza moral, intelectual y emocional del orador. Por ejemplo, los mejores oradores pronunciaban sus sermones con una celeridad 60 por ciento más rápida que el ritmo de una conversación normal, es decir, 500 sílabas por minuto (Merrill, 1988: 64).

Tal como registré en Norogachi y de acuerdo con la etnografía de W. Merrill, los sermones se ejecutaban los domingos en el atrio de la iglesia, posterior a los oficios religiosos católicos, y estaban dirigidos a la población congregada, entre veinte y cien personas. Mientras la concurrencia guardaba silencio, el orador "se mant[enía] de pie [...] e inmóvil excepto por el movimiento de sus labios y el gesto enfático ocasional con su mano. Sus palabras flu[ían] monótonas en una intensa corriente, que aumenta[ba] en tono constante pero casi imperceptiblemente mientras avanza[ba]. La multitud est[aba] atenta pero impasible durante el sermón; solamente si el orador vacila[ba] los oyentes ofrec[ían] aliento gritando: '¡Ten fuerza!' (¡lwérasa!)” (Merrill, 1988: 64). Al final del sermón, la multitud replicaba al unísono, jweé gará júku! (jes buena!). El orador respondía con un arioshibá mateterabá (Gracias, adiós), y la gente contestaba mateterabá. Finalmente, el orador indicaba que la gente podía marcharse.

El tema de los sermones giraba en torno a la descripción de la conducta de Onorúame y de Eyerúame, comprendida como un ejemplo de cómo vivir. En ambos casos, el camino y el caminar fungían como dos referentes para la correcta acción colectiva, tal como muestran los siguientes ejemplos.

\footnotetext{
Sigan el camino de Nuestro Padre y el camino de Nuestra Madre. No estén tristes ni descorazonados. ¿Qué bien hay en estar tristes? ¿Se desalientan Nuestro Padre y Madre mientras van cuidándonos aquí en la tierra? Todos busquen una vida larga. Prosigan vigorosamente otro día, otra noche, otro año. Nuestro Padre y Nuestra Madre nunca faltan un día o un año. Siempre están aquí en la tierra. [Fragmento de sermón recopilado por W. Merrill el 1 de enero de 1987 en el pueblo de Basíhuare (Merrill, 1992 [1988]: 106-107), traducción del autory resaltado mío].

Regresen aquí otra vez el próximo domingo, yo les digo. Nunca dejen de venir aquí a pedir el perdón de Nuestro Padre y Nuestra Madre. Al hacer esto, ustedes siempre caminarán con fuerza. No estén tristes. No sigan a Aquel Que Vive Debajo porque si lo hacen terminarán Ilorando. Sean fuertes y estén contentos dondequiera que vayan. ¿Acaso Aquellos Que Nos Proporcionan La Luz son perezosos al caminar allá arriba? Yo les digo: ellos me cuidan y los cuidan a ustedes. Por eso, tomen aliento y vayan con fuerza. No estén tristes. Vuelvan contentos aquí otra vez el domingo. [Fragmento de sermón recopilado por W. Merrill el 11 de febrero de 1979 (Merrill, 1992 [1988]: 110-111), traducción del autor y resaltados míos].
} 
En estos sermones, la alusión a los caminos no se reduce a una metáfora o a una figura retórica. Los caminos de Onorúame y Eyerúame, El-que-es-Padre y Laque-es-Madre, refieren al transcurso del sol y de la luna en el firmamento y en el horizonte. Estos desplazamientos marcan los ritmos de las actividades agrícolas y rituales y, con ellos, los distintos momentos de movilidad de los rarámuri en la Sierra Tarahumara. Son abundantes las descripciones etnográficas sobre este tema en regiones de barranca (Kennedy, 1970; Levi, 1993; Olivos Santoyo, 2012), en regiones de cumbre (Merrill, 1988; Graham, 1996; Martínez Galván y Naranjo Mijangos, 2012) y en zonas urbanas (Morales Muñoz, 2013; Naranjo Mijangos, 2014; Herrera Bautista, 2013). Además, estos caminos remiten al modo específico para realizar este andar colectivo. La alegría y la fuerza deben caracterizar las acciones, los pensamientos y las palabras de las personas. De lo contrario, la tristeza y la enfermedad se propagarían en los caminos que cruzan los cuerpos, en los senderos celestes de los astros y en los andares que los rarámuri transitan cotidianamente. En los rituales mortuorios (nutéma) se expresa claramente el riesgo colectivo de la tristeza personal. Por tal motivo, durante las nutéma, se exhorta a los deudos y a los presentes a estar contentos, danzar y beber cerveza de maíz (Fujigaki Lares, 2015). Finalmente, estos caminos remiten a la costumbre o al camino de los antepasados (anayáwari boé) que, dicho en palabras de Luisa Bustillos y Antonio Sandoval, matrimonio rarámuri destacado por su labor social en Norogachi: "es como ir caminando por un camino, todo el pueblo rarámuri".

Esta presentación de los sermones es el marco para explicar por qué las narrativas registradas durante el desarrollo inicial de mis pesquisas etnográficas estaban definidas por las condiciones epistemológicas que determiné a través de mis herramientas metodológicas. En otras palabras, al utilizar entrevistas dirigidas y/o abiertas, el diálogo entablado con los rarámuri de Norogachi produjo un material que denominaré mitos. A continuación, expongo un par de ejemplos de este segundo contexto de enunciación, retomados de mis diarios de campo.

\section{FRAGMENTO DE ENTREVISTA 1.}

Entrevistador/Entrevistadora: ¿Sabes alguna historia de cómo empezó el mundo?4

Cervando: Pues sí, sí me contaba mi jefa [mamá]. El mundo se creó, cree la gente pues que se creó por medio de zoquete, porque antes era nomás zoquete, cuando era todavía tibio el mundo, porque muchas veces dicen que se ven las huellas por ahí. Cuando todavía estaba tierna la tierra, entonces se ven huellas de caballo, huellas de gente, hay muchas cosas pues. Estaba todavía tierna [la tierra], todavía no se enfriaba bien y por eso se ven las huellas. [Fragmento de entrevista con Cervando Sandoval en el rancho de Cochérare, Norogachi, 12 de noviembre de 2003. El énfasis es mío]. ${ }^{5}$

Fragmento de entrevista 2.

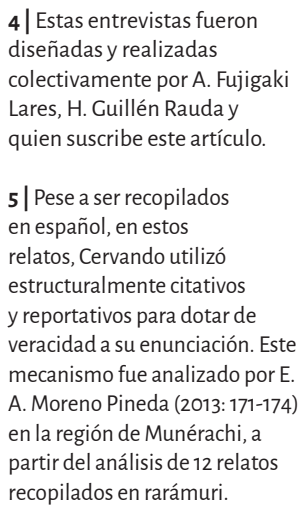

4 | Estas entrevistas fueron diseñadas y realizadas colectivamente por A. Fujigaki Lares, H. Guillén Rauda y quien suscribe este artículo.

5| Pese a ser recopilados en español, en estos relatos, Cervando utilizó estructuralmente citativos y reportativos para dotar de veracidad a su enunciación. Este mecanismo fue analizado por $\mathrm{E}$. A. Moreno Pineda (2013: 171-174) en la región de Munérachi, a partir del análisis de 12 relatos recopilados en rarámuri. 
Entrevistador/Entrevistadora: ¿Sabes alguna historia de cómo empezó el mundo?

Andrea: Cómo empezó el mundo, el primer mundo que se puso bien, [...] se hizo pues el primer mundo que se hizo, se hizo tres mundos al principio, no había gente, no había nada, nada más había puros animales y después se fue haciéndose más y más y se acabó otra vez el mundo. Y luego a los, a los tercer [sic] año hubo otro mundo y luego a los tres años ya, ya vivieron toda la gente, ya vivieron mucha gente, hubo mucha siembra, hubo muchas cosas. El primer mundo se acabó con el agua caliente que hirvió. El segundo le pasó el sol, bajito, bajito le pasó el sol. Y luego, el tercero pus ya es la que vive ahora.

\section{Entrevistador/Entrevistadora: ¿Y los rarámuri?}

Andrea: Los hizo en barro, de barro de ese que se hacen ollas pues, de eso los hizo. Primero hizo el niño, primero hizo el hombre pues, después, ya después dijo no pus' voy a hacerle una compañera, voy a hacer una mujer, y luego le quitó la costilla del hombre, ya ves que casi no tienen costilla el hombre, porque nos prestó las costillas que tenemos a hí también. Y ya después dijo ya tiene compañía ya no va a estar solo. Sí, los hizo de barro y luego ya pues les sopló. [Fragmento de entrevista con Andrea Loya, pueblo de Norogachi, 15 de octubre de 2003].

El material antes expuesto fue recopilado en español y, en menor medida, en rarámuri. Por tanto, conforma un corpus operativo para reflexionar sobre los procedimientos de producción y de transmisión de un conocimiento co-inventado, sensu R. Wagner (1975). Al igual que los rarámuri con los que conversé hasta el 2016, sea en español o en rarámuri, por mitos entiendo los materiales co-inventados entre rarámuri y aquellos que éstos definen como no rarámuri (mestizos, mexicanos, norteamericanos etc). La finalidad de estos materiales es estabilizar los "saberes antiguos" (kite amachíala kiya nirúami) en la escritura alfabética. ${ }^{6}$

Al igual que en el caso del Alto Río Negro documentado por S. Hugh-Jones (2016) y P. Cesarino entre los marubo del Vale do Javari (2011), para los rarámuri, la cantidad y la diversidad de estos materiales son una expresión del interés recíproco que estaría comprometido en la co-invención de estas publicaciones.? Particularmente destaca la intención de los rarámuri en visibilizar su participación como gestores, recopiladores, traductores, enunciadores, editores y compiladores. Más aún, la creciente producción de poesía rarámuri (Makáwi, 2012; Batista, 2011) y de soportes visuales, tales como cómics, videos y videojuegos articulados con esta mitología, son indicativos de este proceso. ${ }^{8}$ Por estas razones, considero que los mitos de/sobre los rarámuri son un vínculo de co-invención de conocimiento entre rarámuri y no rarámuri, es decir, un dispositivo para indigenizar la cultura en el sentido otorgado por M. Carneiro da Cunha (2009: 277-374), que, simultáneamente, oblitera el tiempo al reproducirsey, por tanto, al transformarse (Gow, 1996: 287-296). Reconocer los proyectos de existencia vinculados y expresados en estos materiales conforma un rico campo de reflexión que aún no ha sido explorado en su totalidad.
6| "Saberes antiguos" (kite amachíala kiya nirúami) es el título de un libro de distribución gratuita para las escuelas públicas en el estado de Chihuahua editado por los rarámuri J. Gardea Carcía y M. Chávez Ramírez (1998).

7| Sugiero consultar el acervo bibliográfico en línea de del Instituto Nacional de los Pueblos Indígenas (INPI) en http://201.134.246.21/ cgi-bin/koha/opac-search. pl?q=su:\%22CUENTOS\%20 TARA HUMARAS\%22 [consultado 16 de mayo 2019].

8 | Ver proyectos como 68 voces, 68 corazónes en http://68voces. $\mathrm{mx} /$ tarahumara-el-origen-delos-raramuri-y-los-chabochi [consultado 16 de mayo 2019]. "El origen de los rarámuriy los Chachochi" en https:// vimeo.com $/ 168708547$ [consultado 16 de mayo 2019]. "Cuento mexicano por niños tarahumaras" en https://www.youtube.com/ watch?v=HoK43cgaYDw [consultado 16 de mayo 2019]. "Cawichi" https://www.youtube com/watch?v=80mpVX 3 u6k [consultado 16 de mayo 2019]. Y el videojuego Mulaka producido por NINTENDO ver https://www.lienzo.mx/ mulaka/?lang=es [consultado 16 de mayo 2019]. 'Tepórame', la historia en cómic del héroe rarámuri que peleó contra los españoles en México", El país, 28 de julio de 2019 https://verne elpais.com/verne/2019/07/27/ mexico/1564254769_895842. html?id_externo_rsoc=FB CC\&fbclid=IwARoX9v495y MwkFb4rahQxy YKiREjiRI6 JoEdKyoFfAVmhlcc3b--IV9xw [consultado 6 de agosto 2019]. 
Finalmente, el tercer contexto enunciativo fue destacado recientemente por E. Servín Herrera (2015). De acuerdo con este intelectual y literato chihuahuense, la producción y la transmisión de chabé nilúame natáli ("lo que se piensa antes") entre los propios rarámuri difieren de los consejos y de los mitos. Siguiendo a ese autor, una de las técnicas epistemológicas de los rarámuri para producir y transmitir este saber consiste en la enunciación colectiva. ${ }^{9}$

Un grupo se reúney, a las afirmaciones primarias de cualquier persona, deberán sumarse otras y así, sucesivamente, hasta conformar conjuntamente una narrativa. Por ello, como E. Servín Herrera afirmó, el hecho de estabilizar estos relatos en una versión escrita, como las que presenta a manera de literatura en su libro "Anirúame" (literalmente "lo que fue dicho"), no sólo traduce sino que traiciona la práctica de transmisión y de producción de tal conocimiento. Hecho que este intelectual confirmó cuando, al solicitar a los viejos rarámuri narrar historias en la radio regional, la XETAR, "La voz de la Sierra Tarahumara", 870 de AM, recibió como respuesta diversas resistencias y dificultades. Los rarámuri afirmaban que no sabían "historias", más aún, guardaban recelo ante el contexto de enunciación solitaria en una cabina radiofónica.

En consonancia con lo descrito por E. Servín Herrera, las técnicas y el arte para reír de algunos rarámuri que conocí entre 2002 y 2012 son un ejemplo del contexto de enunciación colectiva. En el invierno de 2003, mis compañeros de campo y yo nos encontrábamos sentados en un círculo de personas bebiendo cerveza de maíz. La broma inició: un hombre afirmó que una mujer tenía sexo con un hombre en un granero, acto seguido, la concurrencia rio, supusimos que la hilaridad se debía a que los presentes conocían a los sujetos involucrados. Sin embargo, la broma apenas comenzaba. Otra persona tomó la voz y con los mismos elementos, pero mediante otra enunciación, repitió lo dicho por la primera persona y dijo: "quien veía las tablas del techo era el hombre, no la mujer". Todos rieron un poco más fuerte y durante más tiempo. Alguien más tomó la palabra y dijo: "quien las miraba era la mujer". Esta dinámica, las risas y la circulación de la bebida, se prolongó durante aproximadamente una hora. En cada intervención, los participantes repetían la misma información sin modificar los elementos iniciales de la historia. ${ }^{10}$

Desde mi perspectiva, la gracia de la broma residía en la forma de producción de la enunciación. Esto es, en hacer una variación mínima de los elementos primarios hasta conformar un acto colectivo, tanto de enunciación como de diversión. Dicho de otro modo, lo gracioso no radicaba exclusivamente en el contenido de la historia, sino especialmente en la manera de comunicarla. Este mecanismo de producción microscópica de la variación es constitutivo de la epistemología rarámuri y, en consecuencia, de la producción de cultura material, de las personas y de las redes sociales que configuran el socius de este pueblo. Sin duda, este es otro rico campo de estudio por explorar que la antropología y la lingüística podrían indagar interdisciplinariamente. $\mathbf{9} \mid$ Estas afirmaciones fueron retomadas de la presentación del libro Anirúame "Lo que fue dicho", del jueves 18 de febrero de 2016 en la XVII Feria Internacional del Libro de Palacio de Minería en la Ciudad de México, a partir de la comunicación personal de Bianca Islas. De manera similar, K. H. Basso (1996: 77) al construir una etnografía del habla entre los apaches, indica que la conversación es una forma de cooperación voluntaria en la cual la enunciación-entendida como la evidencia del pensamiento que ocurre bajo la forma de imágenes que las personas miran en sus mentes - es completada al ser escuchada esto es, al tomar las palabras del hablante y ver las imágene en las mentes de la audiencia.

10| A esto es preciso agregar las traducciones del rarámuri al español que la pareja que nos invitó elaboró para nosotros pues, en aquellas primeras visitas, nuestra comprensión del rarámuri era limitada. 
Hasta este momento, describí tres contextos de enunciación rarámuri sobre "lo que se piensa antes". El camino y el caminar, como guías de acción individual y colectiva, son un asunto central en los consejos y en los mitos revisados. La finalidad de presentar este material, reitero, fue ofrecer un marco general de comprensión para atender al contex to de producción y de transmisión de conocimiento articulado con el acto de caminar, tópico del siguiente apartado y tema central de este artículo.

\section{MOMENTOS ETNOGRÁFICOS: ENTRE LA PALABRA Y EL CAMINAR}

El vínculo entre las experiencias de campo inscritas en un diario etnográfico y el trabajo de sistematización que requiere la escritura, tal como advirtió M. Strathern (2014: 350), están definidos por la continuidad de momentos etnográficos concretos. Las narrativas enunciadas por algunos rarámuri durante las caminatas compartidas y registradas en mis diarios de campo desde el 2003 son los momentos etnográficos que definen mi reflexión en torno al registro territorial de la historia rarámuri. Para ejemplificar dicho vínculo, presento algunos extractos de mis notas de campo para, posteriormente, destacar sus consecuencias.

El día 19 de octubre de 2003, Alejandro Fujigaki, Héctor Guillén y yo caminamos durante dos horas desde el pueblo de Norogachi hacia el rancho de Kocheráre, situado hacia el sur, donde viven aproximadamente diez familias. Visitamos a Cervando Sandoval, hombre de 38 años, de ojos rasgados y una piel cobriza quemada por el sol. Cervando está casado con María, una mujer de 27 años, alta, como de $1.70 \mathrm{~cm}$, delgada, de ojos brillantes y nariz aguileña. Tienen dos hijos. Cuando llegamos a su casa, Cervando y María estaban pizcando, se disponían a comer, nos invitaron. [...]

Después de comer, salimos a caminar. Cervando nos habló de unas pinturas en las piedras y fuimos a conocerlas. Los dos sitios de pintura rupestre se encontraban en la orilla de dos arroyos. Cervando relacionó ambos sitios con el inicio y con el fin de uno de los mundos, en el cual el sol se acercó tanto a la tierra que quemó a la gente. Nos dijo que, en los dos sitios donde había pintura rupestre, había huesos de aquellas personas que se escondieron del sol. Las piedras parecían carbonizadas, por ello, comentó que eso era producto de la vez "que el sol pasó muy cerca de la tierra y chamuscó todo". En el primer sitio nos enseñó un pedazo de un hueso, que no tocó con la mano, sino con un palo. Nos dijo que era colorado, por eso sabía que era de humano, pues los huesos blancos son de animales. [...] Dijo que, debajo de las piedras donde estaban grabados y pintados los diseños, estaba enterrada una señora. Por ese motivo, comentó, ese lugar se llamaba Kochérare que significa: "cuando ya no hay salida, como acorralado". A esa señora, en una ocasión, se le aparecieron tres serpientes y ella subió a una piedra grande, pero las tres víboras comenzaron a dar vueltas alrededor de la piedra. Cervando aquí terminó su relato.

Deacuerdo con el diario de campo de mis colegas, a lo largo del recorrido, Cervando comentó anécdotas e historias relacionadas con los lugares por los que caminábamos. 
ARTículo | María Isabel Martínez Ramírez | El camino y el caminar: fuentes históricas de los

Cuando Cervando era niño, guardaban a limentos en los recovecos de las piedras durante la temporada de invierno para que no se congelaran. En uno de esos nichos rocosos que visitamos, indicó que, alguna vez, encontraron una olla con oro y que también, en ese lugar, refugiaban las chivas cuando nevaba. Pero que, por maldad, una persona había cercado el sitio. Nos contó que por ahí también estaban las antiguas cuevas de los apaches. Que eran personas que venían, como el chabochi [término para referirse a los mestizos locales y a los mexicanos en general], a quitarle las mejores tierras a los rarámuri. Que venían a abusar de ellos. "Se contaba", afirmó Cervando, "que cuando no tenían que comer se comían niños rarámuri, y los burros comenzaban a rebuznar cuando olían lo que cocinaban porque los animales se daban cuenta cuando pasaba eso". Nos dijo también que por ahí estaban las cuevas en las que se escondieron los padres cuando el gobierno los perseguía [durante la Guerra Cristera, 1926-1929]. Su papá, que era muy amigo de ellos, les ayudó a escapar cuando los perseguían.

Durante el trayecto, Cervando narró que trabajó para los mestizos que eran dueños de las tierras sobre las que caminábamos. El pago que recibía era de 150 pesos, más tres comidas, por una jornada de trabajo de siembra. Por cada lugar que pasábamos, contaba algo sobre las personas que vivían o vivieron en Kochérare. Por ejemplo, comentó que la mayoría de las tierras era de los chabochi y que, con el paso del tiempo el precio de las tierras aumentaba. Relató cómo su madre compró unas tierras hace mucho tiempo por dos vacas. Sin embargo, resal tó que ahora el valor de la tierra era monetario.

Estos fragmentos ejemplifican las similitudes y las diferencias entre las narrativas creadas en el contexto de las entrevistas dirigidas, presentadas arriba, y de los relatos generados durante el acto de caminar. En ambos casos, mis colegas y yo fuimos los interlocutores a los cuales se dirigía la producción y la transmisión del conocimiento. Aún así, una de las distinciones más evidentes es que las palabras de Cervando tenían como fin traducir, en el sentido amplio de la palabra, un conocimiento registrado en el territorio que, desde su perspectiva, era relevante para nosotros.

Al igual que en otras ocasiones en las que caminamos con los rarámuri, Cervando compartió un conocimiento sustentado en registros visibles e identificables. Con base en el reconocimiento del hollín de las cuevas y de los huesos humanos, este hombre rarámuri relató parcialmente el mito de origen rarámuri publicado en distintos libros sobre la formación del mundo actual. Este mito de estructura serial relata la configuración del modo de existencia rarámuri mediante la creación y la destrucción de tres mundos. El primero es destruido porque el sol desciende, el segundo por una especie de diluvio o de inundación por agua hirviente, el tercero por la muerte del sol. En cada uno de estos episodios, los rarámuri acometen transgresiones por: a) olvidar cómo hacer como rarámuri y embriagarse sin compartir previamente la cerveza de maíz con Onorúame; b) por actuar como los otros al practicar el canibalismo; o c) por olvidar cómo ser y actuar ignorando la ritualidad y el sacrificio necesarios para Onorúame. De igual manera, a través del reconocimiento de algunos resguardos rocosos, Cervando relató eventos políticos acontecidos a inicios del siglo XX, procesos de reordenamiento 
territorial provocados por la presencia de los no rarámuri y acontecimientos familiares y personales de su vida.

Desde el clásico artículo de E. E. Evans-Pritchard (1939) sobre el tiempo entre los nuer, contamos con una extensa bibliografía antropológica dedicada a comprender este tipo de experiencias y conceptualizaciones temporales. En México, destaca el trabajo de V. Reifler Bricker (1981) sobre la relación entre historia y mitología maya; y los innovadores acercamientos de F. Navarrete Linares (2004) y de R. Lira Larios (2017) en torno a los cronotopos amerindios. Para el caso que nos ocupa, W. Merrill (1992 [1988]: 117) indicó que el marco de los sermones rarámuri era restricto y lineal pues "vinculan a la sociedad contemporánea rarámuri y a su cultura con sucesos que ocurrieron a principio del mundo, pero no contienen alusiones a la reciente historia ni a los numerosos hechos antiguos que los rarámuri relatan en sus cuentos y mitos". Hecho que indudablemente contrasta con los relatos de Cervando. Si bien, el material expuesto podría ser analizado desde estas perspectivas, aquello que me interesa es la relación entre el contexto de producción y de transmisión del conocimiento y las temporalidades presentes en estas narrativas.

Por una parte, Cervando pretendía enseñarnos - recordemos que mis colegas en campo y yo éramos los destinatarios del conocimiento y, por ello, el español fue la lengua de transmisión de este saber - a reconocer los registros visibles que eran una evidencia de eventos pasados (p.e. los restos de hollín, los huesos y las ollas de barro en cuevas). Por otra parte, las temporalidades vinculadas con los registros territoriales identificados eran una expresión de las relaciones concretas que este hombre rarámuri mantenía con su mundo o kawí. De tal manera que el manejo de distintas temporalidades (tiempo del origen, fines del siglo XIX e inicios de siglo XX, vida familiar y personal) y escalas (vida personal, vida familiar, manejo de las tierras del rancho, uso político del territorio y configuración del mundo actual) ubicaba narrativamente a Cervando en una compleja red espaciotemporal que, posiblemente, aglomeraba su conformación como persona. Mediante dicho procedimiento, este hombre construía y compartía su posición enunciativa con nosotros, la cual, simultáneamente, otorgaba validez y autoridad al conocimiento transmitido. El siguiente ejemplo, tomado también de mi diario de campo de 2003, confirma lo dicho.

El martes 17 de noviembre de 2003, conversamos con Bautista Bustillos cerca del Arroyo de la Luna en Secoráchachi, aproximadamente a un día de camino del pueblo de Norogachi. [Bautista era un hombre alto, quizá $1,90 \mathrm{~cm}$, participó en la estructura ritual de semana santa y, por curiosidad, a él le gustaba conversar con nosotros]. El invierno llegaba y, con él, la nieve. Aquella noche dormimos en un resguardo rocoso de aproximadamente cinco metros de altura ubicado en la orilla del arroyo y protegidos por un tronco ardiente que servía de fogata. Bautista narró que: "Los apaches tenían un campamento ahí [señala un cerro ubicado del otro lado del arroyo]. Y todavía existe. O sea, quién sabe qué ponían, tienen redondeados [en círculos] así las piedritas, que tenían...no sé que tenían, 
ARTículo | María Isabel Martínez Ramírez | El camino y el caminar: fuentes históricas de los

pues, según dicen, que usaban como cuero que a todos, con palitos, así tenían, ahí dormían adentro. $Y$ cuando hacíamos canoa [nombre dado a los troncos utilizados para construir una casa] llegaban los apaches así, ellos iban y tomaban el teswino [cerveza de maíz]. No, pues no más se ponían a ver, y pues qué les decían. Se enojaban, porque eran muy bravos, no, luego lo intentaban matar a uno. No, pues se quedaban mirando nada más. Porque se ponían bien ebrios, pues se acababan el teswino y se iban a su campamento allá. Pues no les decían nada, porque tenían miedo de morir. Como hay en Norogachi, ahí en la cumbre, ahi también vivían. Y aquí abajo también vivían. Es que otros aquí en frente y otros vivían acá enfrente. Ahí tienen su cerquito, ahí donde tenían cercado [señala con la mano un perímetro de piedras cubierto de vegetación]. Y de ahí se defendían cuando alguien iba a ... como a pelear con ellos. Ya tenían cercados ahí, y, se ven las cercas, ahí se ve cercado todavía. Está cerquita, ahí cerquita. Es que em Norogachi, ahí están los dos cerritos esos, ya ve que está el cerro de la Cruz y el otro de al lado, como es redondo, es noloa, entonces ya le pusieron Noloachi, pero en rarámuri es Noloachi, Norogachi es en chabochi [español]. Pero noloa, es Noloachi. Porque como que da vuelta el cerrito ese, es redondo y es Noloachi. Sí, por eso le dicen Norogachi, como da vuelta, Norogachi.

Al igual que Cervando, Bautista echó mano de los registros reconocibles en su campo de visión para desplegar narrativamente una red conformada por distintas escalas espaciotemporales y ubicarse en ella-como un rarámuri que se distingue de los apaches y de los mestizos, figuras de alteridad rarámuri. Con ello, Bautista definió su punto de enunciación, así como la validez y la autoridad del conocimiento sobre el nombre del pueblo de Norogachi. Después de esta primera conversación, caminamos con Bautista hacia una ranchería llamada Simúchichi, ya que este hombre rarámuri deseaba mostrarnos unas cuevas con depósitos de cerámica y, como afirmó, "huesos de los antepasados". Comparto algunos fragmentos de mi diario de campo.

Narárachi, se llama así porque unos tarahumares [sic] le prendieron fuego a una cueva en la que vivían los apaches, estos apaches comenzaron a llorar porque pensaron que se iban a quemary por eso ahora el sitio se llama así, nará es llorar".

Bautista hablaba mucho del dinero que hay enterrado en las cuevas, incluso nos pidió un detector de metales. Atribuía ese dinero a la gente que había muerto en la Revolución [1910-1921] y que había dejado su dinero en las cuevas, enterrado. "El problema es que luego también ahí enterraban a la gente y eso era un problema porque si uno se llevaba las cosas luego se enfermaba o no podía dormir". Él pensaba que el olor de las monedas enfermaba a la gente, pero en varias de sus narraciones lo atribuía a los muertos.

"En la guerra cristera, cuando se perseguía a los curas, daban misa en las cuevas y ahí vivían, donde había anayáwari, la gente que andaba con ellos iba a misa. Los curas y las monjas se estaban escondiendo".

Nuevamente, en este material, se manifiesta la particularidad del conocimiento producido al caminar. Para nuestros interlocutores rarámuri, era relevante 
transmitir los procedimientos de producción de conocimiento, antes que saberes concretos por sí mismos - mecanismo que, por ejemplo, estructuraba la información recopilada por medio de entrevistas y, en gran medida, la mitología estabilizada en la escritura. Por ello, el uso del español, por parte de nuestros interlocutores, resultó operativo para dar cuenta del proceso bajo el cual era fundamental reconocer: 1) las cualidades físicas y geográficas de los registros territoriales, 2) su articulación con una amplia red espaciotemporal, 3) la posición del enunciador y 4) la validez del conocimiento compartido. A partir de estas enseñanzas, fue factible introducirnos posteriormente a la historia rarámuri mediante la enunciación de frases muy breves, en español o en rarámuri. Este fue el caso de una caminata que compartimos con Lupe Espino en 2005 hacia Bacóchi. Inesperadamente, este hombre de aproximadamente 45 años, y quien fungió como gobernador rarámuri, se agachó y dijo señalando el suelo: "ven estas huellas de animales en las piedras, es de cuando el mundo se llenó de agua". A través de esta y de otras frases similares, caracterizadas por su capacidad de síntesis, reconocíamos la obviación de los mecanismos epistemológicos para producir y transmitir conocimientos concretos; los cuales fueron explícitos durante nuestras primeras visitas de campo para gradualmente quedar implícitos en la interacción. Para los apaches con quienes trabajó K. H. Basso (1996: 83), esta capacidad evocativa de sustituir una narrativa por un nombre era denominada "speaking with names". Como declararía uno de sus interlocutores: "Nombres-lugares es todo lo que necesitamos para eso, hablar con nombres. Nosotros los fijamos ahí".

Antes de examinar por qué los registros territoriales son una fuente de la historia rarámuri - tema del apartado final y conclusión de este artículo-, expongo dos reflexiones vinculadas a estos momentos etnográficos. Primero, resulta sugerente la analogía entre la enunciación rarámuri producida en el contexto del caminary las propuestas de C. Severi (1996; 2010[2004]) y de P. Cesarino (2011; 2013) dedicadas a dilucidar el paralelismo entre la palabra y la imagen que constituye a las poéticas chamánicas amerindias. La pretensión teórica de esta analogía es mínima, pues el contexto rarámuri difiere de la articulación de las poéticas chamánicas y los registros gráficos elaborados entre los cuna de Panamá o los registros corporales entre los marubo del Vale do Javari en Brasil. Aún así, es interesante reconocer que, para el caso rarámuri, la enunciación difiere de los registros territoriales que, al ser evidencia y generadores de la palabra, funcionan como el soporte material de las narrativas. Aquello que resulta sugerente para la reflexión final de este artículo es que la relación entre la enunciación y los registros territoriales rarámuri contrasta con la lectura de un texto, la ilustración o los indicios a través de los cuales se reconstruye un hecho. Estos registros son un soporte para la producción de la historia rarámuri con una lógica propia, fundamentada en el camino y en el caminar. Este hecho aporta, para dar cuenta de la simetría analítica y política de estos registros, con otro tipo de fuentes, 
tal como S. Hugh-Jones (2016: 176) destacó al examinar la articulación entre las formas gráficas y no gráficas con la oralidad.

Segundo, el régimen de conocimiento al que remiten los momentos etnográficos descritos es análogo al formulado por M. Carneiro da Cunha (2009: 364-367) en torno a los conocimientos tradicionales. Para este régimen, es esencial transmitir los modos de producción de los conocimientos, antes que los conocimientos por sí mismos. En consecuencia, es un régimen abierto al cambio y a la innovación de los contenidos. Sumado a esto, en ambos casos, "los conocimientos tradicionales no son simplemente un corpus estabilizado de origen inmemorial, sino conjuntos duraderos de formas particulares para generar conocimientos" (Carneiro da Cunha, 2009: 364365). Así, antes que los conocimientos, aquello que es antiguo son los procedimientos de producción y de transmisión, es decir, sus criterios de verdad, fuentes y fundamentos de autoridad, así como sus maneras de validación.

Este señalamiento es pertinente para el caso rarámuri. Ya que para garantizar la reproducción de saberes concretos - tal como indican algunos instrumentos internacionales (p.e. Convenio 179 de la Organización Internacional del Trabajo) -, sería indispensable avalar tales procedimientos de producción y de transmisión de conocimiento. En consecuencia, sería fundamental legitimar legalmente las relaciones que los rarámuri mantienen con el kawí y garantizar la reproducción de estos vínculos con la Sierra Tarahumara o su mundo, aquel "place-world" esencial del que hablaba K. H. Basso (1996). Tales relaciones y vínculos son una condición para la existencia y la constitución de cada persona. Pues, como han advertido diversos etnógrafos, crecer en el kawí es indispensable para mantener vínculos de aprendizaje con El-que-esPadre, Onorúame, y, por tanto, para conocer animales, plantas y otros existentes, para experimentar el sueño y la danza, para practicar el silencio y el buen caminar; en fin, para garantizar la continuidad de los proyectos políticos y de existencia de este pueblo (Wyndham, 2009; Salmón, 2000; Fujigaki Lares, 2020).

Por último, todos los registros territoriales enunciados hasta ahora están inscritos en aquello que los rarámuri denominan la piel del mundo o wichimoba (wichi, suelo o piel y moba, encima, en D. Brambila, 1976 [1953]: 598). En este sentido, tal como advertí en la introducción, el derecho a una historia propia derivaría del derecho al territorio y a constituirse como personas y colectivos bajo sus propios términos.

\section{FUENTES DE LA HISTORIA RARÁMURI: A MANERA DE CONCLUSIÓN}

En este artículo describí tres contextos de enunciación rarámuri con el fin de ofrecer un panorama general para reflexionar sobre los relatos relacionados con el camino y con el acto de caminar. De esta manera, en diálogo con el trabajo de K. H. Basso (1996), destaqué una de las particularidades de las narrativas producidas en dicho contexto, esto es, la transmisión de los mecanismos epistemológicos necesarios para 
la producción y la transmisión de conocimientos concretos. Por último, apunté las analogías de estos mecanismos con el paralelismo que estructura algunas poéticas chamánicas en América y con las propuestas en torno al conocimiento tradicional de M. Carneiro da Cunha (2009). A continuación, a manera de conclusión, presento una reflexión del por qué los registros territoriales son una fuente para la historia rarámuri.

Con base en los momentos etnográficos expuestos a lo largo de este artículo, sugiero que las fuentes históricas rarámuri podrían definirse a partir de los procedimientos propios para generar y transmitir información sobre el pasado, y no por contenidos concretos. En consonancia con las apuestas de P. Gow (2001; 2006) sobre la historia amerindia, estos registros territoriales, al igual que las fuentes administrativas y burocráticas generadas por los Estados nación y gobiernos, requieren de un aprendizaje especializado para: 1) ser reconocibles, 2) ser legibles y 3) estar articulados con conocimientos específicos. Como señaló S. Hugh-Jones (2016), los registros territoriales son un expresión, entre otras, de las fuentes históricas. Por tanto, como indicó M. Carneiro da Cunha, estos referentes territoriales son diversos y cambian. De ahí la relevancia de reconocer los mecanismos epistemológicos que, de acuerdo esta autora, permanecen a lo largo del tiempo. Uno de estos mecanismos entre los rarámuri, que aún queda por explorar con mayor detenimiento, es la potencia virtual de estos registros territoriales, capaces de actualizarse en cualquier sitio al interior del kawí.

Reitero que mi interés por producir esta simetría entre fuentes documentales y fuentes nativas, sensu B. Latour, es multiplicarlasy, con ello, hacer proliferar narrativas y los espacios de existencia y de diálogo con las historias amerindias. Sin duda, esta búsqueda por la simetría se produce desde el punto de enunciación de la academia con la intención de evidenciar, para este modo de producción de conocimiento institucionalizado, que la distinción entre ambas fuentes radicaría en el valor otorgado por el analista, antes que en las cualidades o características de la fuente. Por tanto, el afirmar que los registros territoriales rarámuri son fuentes para la historia rarámuri, tal como los documentos burocráticos o administrativos podrían serlo, es un acto epistemológico-y, por tanto, diría E. Viveiros de Castro (2014: 200), político.

Desde la perspectiva rarámuri, la distinción entre sus mitos y su historia radica en los procedimientos de producción y de transmisión de conocimiento, no en los contenidos concretos que sirven como referente. Desde mi lectura, esta es una enseñanza metodológica capaz de revertirse a la historia disciplinar de/sobre los rarámuri. Lo mismo sucede cuando, desde dicha perspectiva rarámuri, reconsideramos las distinciones entre historia oral/historia. Tomemos como caso la historia de la explotación forestal en la Sierra Tarahumara durante el siglo XX.

En la historiografía y la etnografía de la región, es recurrente narrar el proceso de explotación forestal en el siglo XX a partir de fuentes documentales, principalmente las generadas por el gobierno mexicano durante la reforma agraria (Lartigue, 1983; Boyer; 
2017; Sariego Rodríguez, 2015 [2002]). Las marcas de deforestación que caracterizan el entorno territorial de las vías férreas que cruzan la Sierra Tarahumara son el correlato de la cronología del ordenamiento territorial, comandado por la reforma agraria, y una evidencia de los mecanismos de explotación intensiva provocados por los vaivenes del mercado internacional de madera. Por tanto, estas marcas son una fuente adecuada para narrar la historia de la explotación forestal. De acuerdo con F. Lartigue (1983: 29), el Inventario Nacional Forestal de 1965 distinguió cuatro zonas de norte a sur que correspondían geográficamente con la antigüedad de explotación. De forma análoga, de acuerdo con D. A. Salazar González (2009: 18), en el siglo XXI se distinguían dos áreas de actividad de explotación. El área de transformación primaria, constituida por los ejidos serranos que convertían la materia bruta en madera de rollo y el área de transformación secundaria, localizada en los aserraderos de propiedad privada semiurbanos que compraban la madera en rollo para procesarla y manufacturar otros productos.

Bajo el principio de simetría planteado, surgen las siguientes cuestiones: ¿por qué la historia rarámuri, articulada con sus propios registros territoriales, sería distinta a la historia inscrita en las marcas de deforestación de las vías férreas que es reconocible para la antropología y para la historia? ¿Por qué las cuevas, las huellas impresas en las rocas y los propios caminos rarámuri tendrían que calificarse como un tipo de historia menor (mito, historia oral, etnohistoria)? Sugiero que la respuesta reincide en los parámetros impuestos por el analista para definir las fuentes históricas y no en los contenidos o en las cualidades de las fuentes.

En este sentido, propongo que el reconocimiento de los registros territoriales rarámuri como fuentes históricas enriquecería los procedimientos académicos para producir y transmitir conocimientos concretos. Por ejemplo, podríamos problematizar cómo las vías férreas son una fuente pertinente para reflexionar sobre una historia compartida entre los rarámuri, los empresarios, los mexicanos y los funcionarios de gobierno. Y advertir que la definición de estas vías desde la perspectiva rarámuri diferiría de la propuesta por las narrativas de la antropología y de la historia disciplinar. Para los rarámuri, dichas vías serían la expresión de un modo de existencia y de un campo de moralidad de los no rarámuri regidos por el apego al dinero y por la incapacidad de llevar a cabo trabajos colectivos, así como el efecto de las acciones rarámuri provocadas por transitar por los caminos otros (sinú boé) (Fujigaki Lares, 2019). Desde las narrativas antropológicas e históricas, estas vías son el testimonio de procesos económicos ajenos a los individuos y a los colectivos locales, incluyendo a los enunciadores. Así, pese a ser la fuente de una historia compartida, estas vías férreas registran hechos y procesos diferentes. Por una parte, son la evidencia de la ruptura de relaciones parentales entre los rarámuri, los pinos y Onorúame. Y, por otra, son la consecuencia del desgaste ambiental provocado por el desarrollo de la economía regional y global.

Por último, si, desde este punto de vista repensamos la distinción entre historia/etnohistoria, será evidente que todas las historias son historias nativas. El 
carácter étnico de algunas historias nacionales en América es un testimonio de esta afirmación, tal como han documentado y demostrado F. Navarrete Linares (2015: 176) para México, S. Rivera Cusicanqui (2010: 57 y 60) para el caso de Bolivia, J. A. Kelly (2016) para Venezuela y A. Mbembe para Sudáfrica (2011 [1999]). Esto redunda en que el problema para reconocer la existencia de las historias amerindias radica en la "dificultad de adoptar ese otro punto de vista sobre una trayectoria de la que hacemos parte" (Carneiro da Cunha, 1992: 21-22). Mas aún, confirma que dicha dificultad reside en dar cuenta de este "hacer parte" desde el punto de vista de otros pueblos y en admitir que nuestras narrativas y nuestras historias, sus procedimientos de producción y de transmisión, son apenas parte de otras historias o etnohistorias, y que nuestras fuentes son apenas algunas entre muchas otras.

Finalmente, si advertimos los límites de nuestro propio conocimiento (Povinelli, 2013) y la particularidad epistemológica que sostiene nuestros saberes sobre el tiempo y la historia, las fuentes rarámuri serían simétricas a cualquier otra. De tal modo que la carencia que ha definido la mitología, la historia oral, la memoria indígena, la etnohistoria (y otros tantos eufemismos para hablar de las historias amerindias) radicaría en los límites epistemológicos y políticos de los analistas para dar cuenta de otras realidades temporales, y no así en las cualidades o calidades de las fuentes, de las narrativas y de estas otras historias.

Tal como afirmó C. Lévi-Strauss (1999 [1952]) en su célebre Raza y cultura, la historicidad, entendida como la sucesibilidad de una cultura o de un proceso cultural, "no depende de sus propiedades intrínsecas, sino de la situación en la que nosotros nos encontramos en relación a ella, del número y de la diversidad de nuestros intereses en juego con los suyos". Al igual que el viajero de un tren, diría este antropólogo, nuestra posición determina aquello que podemos conocer sobre los otros trenes que, viajando en direcciones distintas y a velocidades desiguales, comparten las vías cercanas de nuestro viaje. Por tanto, reconocer nuestra posición y el recorrido histórico al que pertenecemos podría enriquecer aquella imaginación histórica promovida por F. Navarrete Linares sobre los trayectos en el tiempo de otros pueblos, así como de los trayectos compartidos por nosotros. En última instancia, podríamos multiplicar las historias sobre nuestra relación con estos pueblos $y$, desde la perspectiva de estos pueblos, promover el espacio de existencia y de diálogo con sus propias historias.

María Isabel Martínez Ramírez es antropóloga y actualmente se dedica al estudio de las historias amerindias contemporáneas en México, con énfasis en las perspectivas nativas. En este momento coordina el proyecto Laboratorios de historia contemporánea (PAPIIT IN404220) cuyo objetivo es explorar la potencia aplicada de la disciplina 
histórica y co-producir con personas adscritas a pueblos mayas, cucapá, tarahumaras y mazatecos conocimientos en torno a historias locales.

CONTRIBUCIÓN DE AUTORIA: Agradezco a Alejandro Fujigaki, Julien Mechault y a Hilda Landrove la lectura de manuscrito previo. Las discusiones mantenidas en los seminarios Cosmopolítica y cosmohistoria y Laboratorios de historia contemporánea inspiraron algunas ideas vertidas en el texto. Finalmente, agradezco a los y a las dictaminadoras de este artículo, sus comentarios enriquecieron mis argumentos iniciales.

FINANCIACIÓN: Investigación realizada gracias al Programa UNAM-PAPIIT IN 404220, Laboratorios de historia indígena contemporánea.

\section{REFERENCIAS BIBLIOGRÁFICAS}

Aguilera Madrigal, Sabina. 2011. La faja ralámuli, un entramado cosmológico. México, Instituto de Investigaciones Estéticas/ Instituto de Investigaciones Antropológicas, Universidad Nacional Autónoma de México.

BASSO, Keith H. 1996. Wisdom sits in places. Landscape and Language among the Western Apache, Albuquerque, University of New Mexico Press.

Batista, Lolita. 2011. Cuentos, leyendas y poemas de Lolita Batista. Chihuahua, Consejo Nacional para la Cultura y las Artes/Programa de Apoyo a las Culturas Municipales y Comunitarias [PACMYC]/ Unidad Regional Chihuahua de Culturas Populares e Indígenas/Culturas Populares de Chihuahua, Tradiciones Orales.

BETANCOURT SABATINI, Nuvia de los Ángeles. 2020. Descripción sociolingüística del rarámuri ra'íchari en la escuela comunitaria Benésica Anagupi de Kwechi, municipio de Guachochi, Chihuahua: un estudio de caso. México, Tesis de licenciatura en lingüística antropológica, Escuela de Antropología e Historia del Norte de México.
Bonfiglioli, Carlo. 1995. Fariseosy matachines en la Sierra Tarahumara. México, Instituto Nacional Indigenista.

BONFICLIOLI, Carlo. 2005. "Jíkuri sepawa'ame (La "raspa de peyote"): Una danza de curación en la Sierra Tarahumara. Anales de Antropología, México, vol. 39, nº 2: 151-188.

Boyer, Christopher R. 2017. "Tierras híbridas. Ferrocarriles, comunidades rarámuris y la mercantilización de los bosques chihuahuenses (1900-1910)". In: Escobar Ohmstede, A., Trejo Contreras, A. y Rangel Silva, J.A. (coords.), El mundo rural mexicano en la transición del siglo XIX al siglo XX. México, Casa Chata, pp. 297-313.

BRAMBILA, David. 1976 [1953].

Diccionario rarámuri-castellano

(Tarahumar). México, Buena Prensa.

Carneiro da Cunha, Manuela (org.). 1992. História dos índios no Brasil. São Paulo, Companhia das Letras.

Carneiro Da Cunha, Manuela. 2009. "Cultura" e Cultura: conhecimentos tradicionais e direitos intelectuais". In: Carneiro Da Cunha, M., Cultura com aspas. São Paulo, Cosac Naify, pp. 311-373. 
Cesarino, Pedro de Niemeyer. 2011.

Oniska: Poética do Xamanismo na Amazônia.

São Paulo, Perspectiva/Fapesp.

Cesarino, Pedro de Niemeyer. 2013.

"Cartografias do cosmos: imagem, palavra e conhecimento entre os Marubo". Mana,

Rio de Janeiro, vol.19, $\mathrm{n}^{\circ}$ 3: 437-471.

DE LA PARRA AGUILAR, Daniela.

2012. "Diversidad lingüística en

la Sierra Tarahumara". In: OLIVOS

SANTOYO, N., et. al. (coords.), Los

pueblos indígenas de Chihuahua. Atlas

etnográfico. México: Instituto Nacional de

Antropología e Historia, pp. 273-291.

Evans-Pritchard, Edward Evan. 1939.

"Nuer time reckoning". Africa: Journal

of the International African Institute,

Cambridge, vol. 12, $\mathrm{n}^{\circ}$ 2: 189-216.
LARES, Alejandro Fujigaki. La disolución de la muerte y el sacrificio. Contrastes de las máquinas de transformaciones y mediaciones de los rarámuri y los mexicas. México, Tesis de doctorado en antropología. Instituto de Investigaciones Antropológicas, Universidad Nacional Autónoma de México.

LARES, Alejandro Fujigaki. 2019. “Caminos que bifurcan y multiplican mundos.

Alteridades | identidades entre los rarámuri de Chihuahua". In: MARTÍNEZ RAMÍREZ, M.I., FUJICAKI LARES, A. y BONFICLIOLI, C. (eds.), Reflexividades metodológicas. Estudios de caso de México y Brasil. México, Universidad Nacional Autónoma de México, pp. 357-398.

LARES, Alejandro Fujigaki. 2020. “Caminos rarámuri para sostener o acabar el mundo. Teoría etnográfica, cambio climático y Antropoceno". Mana, vol. 26, nº 1: 1-35.

Recibido el 7 de agosto de 2019. Aceptado el 8 de diciembre de 2020 . 